\title{
STREM-1 IS ELEVATED IN CYSTIC FIBROSIS AND CORRELATES WITH PROTEASES
}

DL Forrester *1, HL Barr ${ }^{1}$, A Fogarty ${ }^{2}$, A Knox $^{1}$

${ }^{1}$ Division of Respiratory Medicine, Clinical Sciences Building, University of Nottingham, City Hospital Campus, Nottingham, NG5 1PB. ${ }^{2}$ Division of Public Health \& Epidemiology, Clinical Sciences Building, University of Nottingham, City Hospital Campus, Nottingham, NG5 1PB.

${ }^{*}$ Corresponding author:

Douglas L Forrester,

Division of Respiratory Medicine, Clinical Sciences Building,

University of Nottingham,

City Hospital Campus,

Nottingham,

NG5 1 PB.

Tel:01158231712

fax: 01158231946

doug.forrester@nottingham.ac.uk

Funded by Nottingham University Hospitals Charitable Trust.

DLF is supported by Wellcome Trust Fellowship WT088614

Keywords: inflammation, MMPs, biomarkers

Running title: STREM-1 is elevated in cystic fibrosis 


\section{Abstract}

Background: sTREM-1 (soluble triggering receptor expressed on myeloid cells-1) is a novel inflammatory marker that may be of clinical use in cystic fibrosis (CF). Dysregulation of the TREM pathway has been demonstrated in other inflammatory diseases and modulation in animal models has therapeutic benefit. We hypothesised that STREM-1 could act as a biomarker of disease in cystic fibrosis.

Methods: Plasma from seventeen patients with CF (stable and pre and post pulmonary exacerbation) and eight healthy volunteers was analysed for STREM-1 and proteases (matrix metalloproteinase-8 (MMP-8), MMP-9 and human neutrophil elastase HNE).

Results: STREM-1 levels were elevated in stable CF subjects compared to controls (148 $\mathrm{pg} / \mathrm{ml}(130-160)$ [median(IQR)] vs.87(55-118) $(\mathrm{p}<0.01))$ but were not further increased during pulmonary exacerbation nor decreased after antibiotic treatment in CF. Protease levels were increased in CF plasma compared to controls: MMP-8 $=$ $3.1 \mathrm{ng} / \mathrm{ml}(1.5-7.6)$ vs $0.3(0.18-0.53)(p<0.01)$ (Wilcoxon)); MMP-9 = 170ng/ml $(124-$ $282)$ vs $49(39-90)(p<0.01) ; \mathrm{HNE}=30.2 \mathrm{ng} / \mathrm{ml}(22.7-30.9)$ vs17.5 $(11.2-22.2)(p<0.05)$. STREM-1 correlated positively with protease levels $\ln M M P-8 r^{2}=0.55(p=0.08)$, InMMP$9 r^{2}=0.61(p<0.05), \ln H N E r^{2}=0.35(p<0.05)$.

Conclusions: STREM-1 is constitutively elevated in CF and positively correlates with protease levels. Modulation of this pathway may be of therapeutic benefit to patients with CF. 


\section{Introduction}

Cystic Fibrosis (CF) lung disease is characterised by airway inflammation and infection although the mechanism of initiation and propagation remains unclear ${ }^{1,2}$. Airway sampling reveals high levels of inflammatory mediators and abundant neutrophils ${ }^{3,4}$. These neutrophils and those found in the systemic circulation are abnormal in cytokine production and response to stimuli ${ }^{5-7}$.

Triggering receptor expressed on myeloid cells-1 (TREM-1) is an activating surface receptor expressed at high levels on neutrophils infiltrating tissues infected with extracellular bacteria ${ }^{8}$. Activation of TREM-1 amplifies the inflammatory response - a key step in the development of systemic sepsis. Several studies in non-CF pulmonary, sepsis and systemic diseases have implicated a role for TREM-1. For example, a soluble portion of the surface bound receptor (sTREM-1) is shed/cleaved from activated phagocytes and elevated levels of this protein are found in the circulation of patients with sepsis and the lower airways of those with in ventilator associated pneumonia $^{9,10}$. Furthermore sTREM-1 is of prognostic value in patients with community acquired pneumonia ${ }^{11}$ and is positively correlated with severity of disease in patients with COPD ${ }^{12}$. Blockade of the TREM-1 pathway in animal studies provides protection against sepsis even after the initiating event ${ }^{13}$ suggesting it may be a possible therapeutic target.

We hypothesised that pulmonary inflammation in CF would lead to increased STREM1 in circulating blood and that STREM-1 would be an easily measured surrogate measure of disease activity. Matrix metalloproteinases (MMPs) have been 
demonstrated to cleave TREM from human stimulated monocytes ${ }^{14}$ and have been shown to be elevated in airway secretions ${ }^{15}$ and associated with exacerbations in $\mathrm{CF}^{16}$. We also hypothesised that MMPs may be responsible for cleaving sTREM-1 from the surface of cells in CF in vivo and explored the association of sTREM-1 and MMPs in patients with $\mathrm{CF}$ and healthy control subjects. 


\section{Materials \& Methods}

Venous blood samples were obtained from seventeen adults diagnosed with CF (on the basis of a known CF genotype and/or a positive sweat test chloride $>60 \mathrm{mml} / \mathrm{L}$ ) and eight healthy controls.

Venous samples were obtained from eight patients requiring intravenous antibiotics for a physician defined pulmonary exacerbation. An initial blood sample was taken prior to administration of the first dose of intravenous antibiotics and again at fourteen (+/-two) days. A 'stable' CF group consisted of nine patients who had no symptoms of exacerbation for at least four weeks. Healthy controls consisted of eight volunteers who had no known systemic disease and no coryzal symptoms (demographics in Table 1). The local Research Ethics Committee approved the study (REC ref. No. 08/H0406/110) and all participants gave informed consent. 
All blood samples were processed within thirty minutes of harvesting and plasma stored at $-80^{\circ} \mathrm{C}$ prior to analysis. Analysis for STREM-1 and proteases was performed in triplicate using commercially available R\&D Systems Quantikine ELISA kits.

Statistical analysis was performed using Stata v11 (Texas). Data were inspected for normality both visually and using the Shapiro-Wilk test. Where values were not normally distributed, data were log transformed or non-parametric tests were performed. A p value $<0.05$ was considered significant. In the statistical analysis of plasma STREM-1 levels, we initially compared healthy controls to individuals with stable CF. We went on to compare individuals with stable CF to those who were experiencing an exacerbation both at initiation and after antibiotics separately. 


\section{Results}

sTREM-1 levels are elevated in stable CF subjects compared to controls but are not increased during CF exacerbations nor decreased after antibiotic treatment in CF

The levels of STREM-1 found in stable CF patients were significantly higher than that in healthy controls $148 \mathrm{pg} / \mathrm{ml} \quad(130-160)$ [median(IQR)] vs.87(55-118) ( $p<0.01$ (Wilcoxon)). The sTREM-1 levels in CF patients during an exacerbation were also significantly higher than healthy controls before treatment $142 \mathrm{pg} / \mathrm{ml}$ (108-177) $\mathrm{p}<0.05$ and after treatment $130 \mathrm{pg} / \mathrm{ml}(100-193) \mathrm{p}<0.05$ but not significantly different to stable CF patients. The levels did not change significantly (paired Wilcoxon) after systemic antibiotic therapy $142 \mathrm{pg} / \mathrm{ml}$ (108-177) vs. 130(100-193) see Fig 1. sTREM1 was not found to be correlated with $\mathrm{FEV}_{1}$ nor was change in STREM-1 correlated to change in $\mathrm{FEV}_{1}$ during the treatment of exacerbations. 


\section{Protease levels are increased in CF serum compared to controls}

Protease levels in the stable CF group were significantly higher than in the control group of healthy subjects MMP-8 $=3.1 \mathrm{ng} / \mathrm{ml}(1.5-7.6)$ vs. $0.3(0.18-0.53)(p<0.01)$ (Wilcoxon)); MMP-9 = 170ng/ml (124-282) vs. 49 (39-90) $(p<0.01)$ (Wilcoxon); HNE = $30.2 \mathrm{ng} / \mathrm{ml}(22.7-30.9)$ vs17.5 (11.2-22.2) ( $p<0.05)$ (Wilcoxon) see Fig 2.

\section{sTREM-1 correlates with protease levels}

The relationship of STREM- 1 and proteases in the nine stable patients with CF and the eight healthy controls group was explored. Data from the Healthy Control and Stable Cystic Fibrosis groups were combined to look at the physiological correlation between the two molecules. (We combined the groups as numbers were insufficient to look at either group alone with any statistical power.) The data for protease levels were log transformed. There were correlations between MMP-9, HNE and STREM-1 (In MMP-8 $r^{2}=0.55(p=0.08)$, InMMP-9 $r^{2}=0.61(p<0.05)$, InHNE $\left.r^{2}=0.35(p<0.05)\right)$ see Fig3. The levels of STREM-1 and proteases were not correlated in the samples taken from patients suffering exacerbations. 



\section{Discussion}

This study demonstrated a constitutive elevation of an important inflammatory regulator (sTREM-1) in the plasma of CF patients. There was no significant increase in the level of STREM-1 during a pulmonary exacerbation and treatment with intravenous antibiotics did not significantly reduce levels. The data also show a positive correlation between STREM-1 and plasma proteases.

The study had a number of limitations. This was a small pilot study powered to show a difference in levels of sTREM-1 between healthy controls and patients with CF so the further conclusions, notably negative ones such as the impact of antibiotics on levels of sTREM-1 need to be interpreted cautiously. The healthy controls were not age and sex matched to the CF groups and smoking histories from participants were not taken. A further confounding factor may be the medications taken by patients with CF that may directly impact on the STREM-1 pathway ${ }^{17}$. The difficulty of defining an exacerbation in CF is a recurring topic and in this study we used the pragmatic approach of a physician-based diagnosis as this is applicable to standard clinical practice. A further limitation to interpretation is the complex nature of protease/antiprotease relationships and active and inactive isoforms thus our use of the measurement of total protease level via ELISA rather than a more complex methodology to allow measurement of activity may not provide the whole picture.

This is the first study to demonstrate an elevation of STREM-1 in the plasma of patients with CF compared to healthy controls. There are very limited data on STREM-1 in lung secretions in CF. A small pediatric study compared levels of sputum sTREM-1 in 
patients with CF ( $n=17)$ to patients with HIV-related bronchiectasis $(n=26)$ and found the sputum levels of STREM-1 to be significantly higher in the HIV-related bronchiectasis group. Although this study analysed sputum rather than serum sTREM1 , it also found no significant elevation of STREM-1 associated with pulmonary exacerbations in the CF group $(n=6) .^{22}$ This mimics the findings of Radsak and colleagues who demonstrated an increase in the levels of STREM-1 in the sera of patients with COPD that was correlated to disease severity ${ }^{12}$ and the demonstration of its utility as a prognostic indicator in community acquired pneumonia ${ }^{11}$. The mechanism behind this increase in STREM-1 may be through a number of processes: an increase in the number of phagocytic cells from which STREM-1 is cleaved, an increase in expression of TREM-1 on the surface of these cells, an increase in the cleavage from the cells, a decrease in binding of sTREM-1 or an alteration in the metabolism or excretion of sTREM-1. Patients with CF have increased numbers of inflammatory cells in the lungs from which STREM-1 may be cleaved and also have increased levels of MMPs in their airways ${ }^{18}$ and serum ${ }^{16}$.

We also demonstrate a positive correlation between MMPs and STREM-1 in stable subjects. This finding was not seen in patients undergoing an exacerbation. As a pilot study, while the data are limited and associations may be a chance finding, the observations are worthwhile considering in the context of the literature.

The association reported here contrasts with the in-vitro work of del Fresno and colleagues. This group explored the nature of TREM-1 expression on monocytes harvested from patients with CF (and COPD) and noted that TREM-1 was down- 
regulated in comparison with monocytes from healthy volunteers and there was also a failure of the CF derived cells to up-regulate TREM-1 in response to challenge with lipopolysaccharide $(\mathrm{LPS})^{19}$. However, when levels of serum sTREM-1 from patients with CF $(n=20)$ were compared with healthy controls no significant differences were found. The levels of sTREM-1 seen in the healthy controls in the del Fresno group appear to be higher than observed in this study and the reason for this is unclear. Control groups in previous studies have had varying levels of circulating STREM-1 and those seen in our study are similar to non bacteremic patients in a pneumonia study $(n=11)^{20}$ and patients with inflammatory bowel disease $\mathrm{e}^{21}$.

MMPs have been shown to cleave TREM-1 from monocytes in vitro ${ }^{14}$ and our data support this as a putative mechanism with higher levels seen in CF compared to healthy controls. MMPs have been shown to be found in elevated concentrations and activity in airway secretions from patients with CF. The relative activity of MMPs is a balance between the protease and tissue inhibitor which has also been shown to be dysregulated in exacerbations. ${ }^{23}$ This dysregulation has been shown to be attenuated by a reduction in proteolytic activity when MMP inhibitors are added to CF sputum. ${ }^{24}$ The close relationship demonstrated between STREM-1 and MMPs in this clinical in vivo setting supports the in vitro observations of Gomez-Pina et $\mathrm{al}^{14}$ that an inhibitor of MMPs (Ilomostat) can block the removal of TREM-1 from monocytes stimulated with LPS.

Animal studies of infection have shown promise in therapeutic manipulation of the TREM-1 pathway. A synthetic peptide derivative of sTREM-1 used in P.aeruginosa 
pneumonia in rats attenuated the inflammatory response not only in the lung but at a systemic level (reduction in TNF- $\alpha$ and IL-1 $1 \beta)^{25}$. This improved survival (compared with TNF- $\alpha$ blockade which decreased survival) suggesting that this is an uncontrolled, detrimental inflammatory response. Murine studies have also shown blockade of the TREM-1 pathway (by a fusion protein of murine sTREM-1 extracellular domain and human immunoglobulin-gamma Fc portion [msTREM-1/IgG1]) provides protection against sepsis even after the initiating event ${ }^{13}$.

In summary, these suggest dysregulation of the TREM-1 pathway in CF. While these data suggest the role for STREM-1 as a biomarker of disease status in CF is limited, the constitutive elevation suggests this pathway as a potential future therapeutic target. Whether the constitutive elevation is due to increased cleavage of TREM from the cell surface through elevated protease levels or through increased trafficking of TREM to the cell surface is unclear. Inhibition of the MMPs which cleave sTREM-1 from neutrophils may also be worth exploring as a therapeutic target. 


\section{References}

1. Armstrong DS, Grimwood K, Carlin JB, Carzino R, Gutièrrez JP, Hull J, Olinsky A, Phelan EM, Robertson CF, Phelan PD. Lower airway inflammation in infants and young children with cystic fibrosis. American journal of respiratory and critical care medicine. 1997 October;156(4 Pt 1):1197-1204.

2. Stoltz DA, Meyerholz DK, Pezzulo AA, Ramachandran S, Rogan MP, Davis GJ, Hanfland RA, Wohlford-Lenane C, Dohrn CL, Bartlett JA, et al. Cystic fibrosis pigs develop lung disease and exhibit defective bacterial eradication at birth. Science translational medicine. 2010 April 28;2(29):29ra31.

3. Konstan MW, Hilliard KA, Norvell TM, Berger M. Bronchoalveolar lavage findings in cystic fibrosis patients with stable, clinically mild lung disease suggest ongoing infection and inflammation. American journal of respiratory and critical care medicine. 1994 August;150(2):448-454.

4. Sagel SD, Sontag MK, Wagener JS, Kapsner RK, Osberg I, Accurso FJ. Induced sputum inflammatory measures correlate with lung function in children with cystic fibrosis. The Journal of pediatrics. 2002 December;141(6):811-817.

5. Corvol H, Fitting C, Chadelat K, Jacquot J, Tabary O, Boule M, Cavaillon J$\mathrm{M}$, Clément $\mathrm{A}$. Distinct cytokine production by lung and blood neutrophils from children with cystic fibrosis. American journal of physiology Lung cellular and molecular physiology. 2003 June;284(6):L997-1003.

6. Adib-Conquy M, Pedron T, Petit-Bertron A-F, Tabary O, Corvol H, Jacquot $\mathrm{J}$, Clément $\mathrm{A}$, Cavaillon $\mathrm{J}-\mathrm{M}$. Neutrophils in cystic fibrosis display a distinct gene expression pattern. Molecular medicine (Cambridge, Mass.). 2008;14(12):36-44.

7. Petit-Bertron A-F, Tabary O, Corvol H, Jacquot J, Clément A, Cavaillon J$\mathrm{M}$, Adib-Conquy $\mathrm{M}$. Circulating and airway neutrophils in cystic fibrosis display different TLR expression and responsiveness to interleukin-10. Cytokine. 2008 January;41(1):54-60.

8. Bouchon A, Facchetti F, Weigand MA, Colonna M. TREM-1 amplifies inflammation and is a crucial mediator of septic shock. Nature. 2001 April 26;410(6832):1103-1107.

9. Gibot S, Cravoisy A, Lévy B, Béné M-C, Faure G, Bollaert P-E. Soluble triggering receptor expressed on myeloid cells and the diagnosis of pneumonia. The New England journal of medicine. 2004 January 29;350(5):451-458.

10. Gibot S, Kolopp-Sarda M-N, Béné MC, Cravoisy A, Lévy B, Faure GC, Bollaert P-E. Plasma level of a triggering receptor expressed on myeloid cells1 : its diagnostic accuracy in patients with suspected sepsis. Annals of internal medicine. 2004 July 6;141(1):9-15. 
11. Tejera A, Santolaria F, Diez M-L, Alemán-Valls M-R, González-Reimers E, Martínez-Riera A, Milena-Abril A. Prognosis of community acquired pneumonia (CAP): value of triggering receptor expressed on myeloid cells-1 (TREM-1) and other mediators of the inflammatory response. Cytokine. 2007 June;38(3):117-123.

12. Radsak MP, Taube C, Haselmayer P, Tenzer S, Salih HR, Wiewrodt R, Buhl $R$, Schild $H$. Soluble triggering receptor expressed on myeloid cells 1 is released in patients with stable chronic obstructive pulmonary disease. Clinical \& developmental immunology. 2007;2007:52040.

13. Wang F, Liu S, Wu S, Zhu Q, Ou G, Liu C, Wang Y, Liao Y, Sun Z. Blocking TREM-1 signaling prolongs survival of mice with Pseudomonas aeruginosa induced sepsis. Cellular immunology. 2012;272(2):251-258.

14. Gómez-Piña V, Soares-Schanoski A, Rodríguez-Rojas A, del Fresno C, García F, Vallejo-Cremades MT, Fernández-Ruiz I, Arnalich F, Fuentes-Prior P, López-Collazo E. Metalloproteinases shed TREM-1 ectodomain from lipopolysaccharide-stimulated human monocytes. Journal of Immunology. 2007 September 15;179(6):4065-4073.

15. Sagel SD, Kapsner RK, Osberg I. Induced sputum matrix metalloproteinase- 9 correlates with lung function and airway inflammation in children with cystic fibrosis. Pediatric pulmonology. 2005 March;39(3):224232.

16. Roderfeld M, Rath T, Schulz R, Seeger W, Tschuschner A, Graf J, Roeb E. Serum matrix metalloproteinases in adult CF patients: Relation to pulmonary exacerbation. Journal of cystic fibrosis : official journal of the European Cystic Fibrosis Society. 2009 September;8(5):338-347.

17. Tong J, Liu Z-C, Wang D-X. Azithromycin acts as an immunomodulatory agent to suppress the expression of TREM-1 in Bacillus pyocyaneus-induced sepsis. Immunology letters. 2011 August 30;138(2):137-143.

18. Power C, O'Connor CM, MacFarlane D, O'Mahoney S, Gaffney K, Hayes $J$, Fitzgerald MX. Neutrophil collagenase in sputum from patients with cystic fibrosis. American journal of respiratory and critical care medicine. 1994 September;150(3):818-822.

19. del Fresno C, Gómez-Piña V, Lores V, Soares-Schanoski A, FernándezRuiz I, Rojo B, Alvarez-Sala R, Caballero-Garrido E, García F, Veliz T, et al. Monocytes from cystic fibrosis patients are locked in an LPS tolerance state: down-regulation of TREM-1 as putative underlying mechanism. PloS one. 2008;3(7):e2667.

20. Ruiz-González A, Esquerda A, Falguera M, Abdulghani N, Cabezas $P$, Bielsa S, Porcel JM. Triggering receptor (TREM-1) expressed on myeloid cells predicts bacteremia better than clinical variables in community-acquired pneumonia. Respirology (Carlton, Vic.). 2011 February;16(2):321-325. 
21. Park JJ, Cheon JH, Kim BY, Kim DH, Kim ES, Kim TI, Lee KR, Kim WH. Correlation of serum-soluble triggering receptor expressed on myeloid cells-1 with clinical disease activity in inflammatory bowel disease. Digestive diseases and sciences. 2009 July;54(7):1525-1531.

22. Masekela R, Anderson R, de Boeck K, Vreys M, Steel HC, Olurunju S, Green RJ. Expression of soluble triggering receptor expressed on myeloid cells- 1 in childhood CF and non-CF bronchiectasis. Pediatric pulmonology. 2015 April;50(4):333-339.

23. Gaggar A, Li Y, Weathington N, Winkler M, Kong M, Jackson P, Blalock JE, Clancy JP. Matrix metalloprotease-9 dysregulation in lower airway secretions of cystic fibrosis patients. American journal of physiology Lung cellular and molecular physiology. 2007 July;293(1):L96-L104.

24. Gaggar A, Jackson PL, Noerager BD, O'Reilly PJ, McQuaid DB, Rowe SM, Clancy JP, Blalock JE. A novel proteolytic cascade generates an extracellular matrix-derived chemoattractant in chronic neutrophilic inflammation. Journal of Immunology. 2008 April 15;180(8):5662-5669.

25. Gibot $S$, Alauzet $C$, Massin F, Sennoune N, Faure GC, Béné M-C, Lozniewski A, Bollaert P-E, Lévy B. Modulation of the triggering receptor expressed on myeloid cells-1 pathway during pneumonia in rats. The Journal of infectious diseases. 2006 October 1;194(7):975-983. 
Table 1. Baseline participant demographic data

$\begin{array}{cccc} & \text { Control } & \text { "Stable"CF } & \text { “Exacerbating"CF } \\ \text { Age } & 31.5 & 28.0 & 25.0 \\ \text { (Median, IQR) } & (30.0-35.3) & (27.0-31.0) & (21.3-28.3) \\ \text { Male/female } & 4 / 4 & 5 / 4 & 3 / 5 \\ \text { Weight } & 67.5 & 59.0 & 58.0 \\ \text { (Median, IQR) } & (58.8-76.3) & (51.0-65.0) & (54.8-66.5) \\ \text { FEV L/min } & & 1.96 & 1.64 \\ \text { (Median, IQR) } & & (1.43-2.53) & (1.27-2.26) \\ \text { FEV1 \%pred } & & (45-72) & 41 \\ \text { (Median, IQR) } & & & (36-62)\end{array}$


Figure 1. Plasma levels of sTREM-1 are increased in Cystic Fibrosis but do not vary during pulmonary exacerbation $\left({ }^{* *}=p<0.01\right)$

Figure 2. Plasma MMP-8, MMP-9 \& Human neutrophil elastase are higher in Cystic Fibrosis than Healthy Controls $\left({ }^{*}=p<0.05,{ }^{* *}=p<0.01\right)$

Figure 3 Plasma MMP-8, MMP-9 \& Human neutrophil elastase vs. sTREM-1 in stable Cystic Fibrosis and Healthy Controls Open circles are healthy controls; closed circles are stable Cystic Fibrosis 